\title{
Safety Management for Foreigners to Prevent and Mitigate the Damage Caused by Traffic Accidents
}

\author{
Dr. Patcharaphong Akarametagul \\ School of Administrative Studies, Maejo University, Chiang Mai, Thailand \\ dr.patcharaphong59@gmail.com
}

\begin{abstract}
This research study the characteristics and causes of traffic accidents with foreigners, problem solving before and after foreigners are involved in accidents and Safety management for foreigners in order prevent and mitigate damaged caused by accidents. This research employ both qualitative and quantitative research methods. Qualitative research data consist of shops entrepreneur, hotel, factories, tourist foreigners, migrant workers and safety management practitioners. Structured interviews was used as an instrument and they were analyzed using content analysis. While Quantitative data consist of foreigners road accident case in Chiang Mai during the year 2014 - 2017. Recorded cases was used as instrument, statistic used include frequency and percentage. The result of the study shows that, the characteristics and causes of traffic accidents to foreigners $2014-2017$ are mostly male. However, road environment is not the main factor of accident but the main causes are driving behavior not knowing the traffic rules or warning signs accompanied by negligence are causing an accident. In terms of problem solving before and after foreigner involvement in accident shows the accident are been reported and notified within 1-3 hours after the incident, but there are also delays in reporting and litigation. This may lead to unfairness because the foreigners do not know how to deal with the situation. Guidelines for the prevention and mitigation of damages caused by traffic accidents for foreigners should be join together such as the driver, the vehicle, road environment. The driver should be force to practice according to traffic laws, traffic laws should be updated and violators should be serious punished. Including community participation in a coordinated manner in order to serve as coordinator or representative to help foreigners by enhance them to understand how to present their selves to foreigners, help communicate with foreigner when they get involved in accident and also at as a mediator in order to bring justice.
\end{abstract}

Keywords: Management guidelines; Traffic accident; Foreigner

\section{Introduction}

The service industry is nowadays playing an important role in the economies of many countries around the world. However, tourism is been deemed as service industry in competing countries just like Thailand, that has developed to be fit for such competitions. (Ministry of Tourism and Sports, 2017) Thailand is a unique country for sustainable tourism management of up to $70 \%$ according to international criteria and there are extensive promotion of creative tourism, which focuses tourist experience in their learning of cultural heritage and the creating of lively bond between tourists and local people in the tourism area. Therefore, result in the creation of good and memorable impression. According to statistics of tourists, that visits Thailand from January to 
September 2017. (Ministry of Tourism and Sports, 2017), found that there were more than $26,100,782$ tourists, however, generate and income of 1,330,058.98 million baht. The top three tourists that visits Thailand were Chinese, Malaysian and Korean tourists.

The above-mentioned tourism situation has resulted in economic development of the country. This is explicitly seen from the structural change of the labor market and production structure that is aimed at producing for export purposes in order to replace import of goods. The occurred changes warrant the country to seek and demand for more labor force. However, most of the labor force in Thailand are migrants. According to current statistics of September from Foreign workers Administration Office Employment Department (2017) found that they were 1,788,964 migrant foreign workers whom are been allowed to work throughout the Kingdom. The migrant workers are from the following three countries Myanmar, Lao, and Cambodian. From the aforementioned figures, illegal migrants were not included and there is tendency for continuous increase in figures.

Moreover, it can be seen that development of tourism and economic sector has resulted in high increase of tourists and foreign workers. However, in this research work, the researcher classify both tourists and foreign workers as "foreigners". Living in an area that is not one's homeland makes it necessary for foreigners to know more about social laws and regulation, especially traffic laws. Due to recent situations, it can be seen that foreigners often get themselves involve in traffic accidents and the causes are from not knowing traffic rules, physical condition of their body, carelessness, vehicles conditions, road conditions, etc. Thailand is been classified as the second country with high rate of road accidents in the world, second only to Namibia. In addition, Daily News (2017) reports the death toll statistics from road accidents all over the world at an average of 18 people per 100,000 people in the world each year. The accidents has nearly 1 million injuries per year and more than 6,000 people are been disabled per year. Especially, during important festivals such as the New Year festival and Songkran festival which has a two times accident rate more than normal days of the year. However, this rate reflects the problems pertaining to safety issues that needs to be addressed and corrected according to World Health Organization (2016) said there is a high rate of foreigners accidents in Thailand and this might be one of the reasons different countries are alerting their citizens in terms of accidents when travelling to Thailand.

According to Sitmonoh et..al (2012) they stated that traffic accidents is an incident that happened unexpectedly, but can be prevented. It is caused by traveling on the road, it involved pedestrians, motorists and all types of vehicle passengers. There are important factors or causes that result to traffic accident such as; personal factor, vehicle and environment. From the study on these three important major factors, which result to traffic accidents (Interrisk Asia, 2017): Personal factors refer to the driver, the passenger, and the pedestrian. Accident, which occurs as a result of personal factors, are up to 43, 000 per year, or 67 percent of the total accidents. The causes of these accidents are negligence, lack of knowledge, not respecting traffic rules. Including lack of self-consciousness in society. Vehicle factor that cause traffic accidents were due to the lack of care and correct maintenance of the vehicle. Including vehicles that do not 
meet safety standards, no inspections were made on the vehicle before driving. Accidents, which occurs as a result of this factor are up to 11,000 per year, representing 17 percent of the total accidents which occurs. These accidents happened because the vehicle were not properly check before departure or some unexpected occurrence during travelling such as stepping on stones, irons, unwanted matter sticking to the underneath of the car etc. Environmental factors is another important factor that causes accidents during driving, Accidents caused by environmental factors are up to 2, 000 per year, or accounted for 3 percent of the total causes. Most of the factors are from weather conditions and visibility that makes it difficult to see, such as rain, slippery roads, dark roads, which can lead to accidents.

Therefore, we can see these factors are related to each other, prevention or correction of problems pertaining to traffic accidents especially Personal factors can be seen from the development that occurred. Both road environment, side lanes, signs, traffic signs. However if individual lacks knowledge and careless, accidents can happen easily. Including the lack of knowledge when foreigners are involved in an accident. They are always cheated and they never received the justice they deserved.

In terms of management approach to prevent and mitigate the damage caused by traffic accidents, the researcher considers that several measures needs to be taken together systematically. Hereby, the researcher presents three standard measures as Morley et..al (2017) said Traffic engineering measures is appropriate engineering modifications based on facilities and technology. Design to reduce accidents, including gathering data that relates to the characteristic and causes of accidents. Including data of road where frequent accident occur as a guideline for improvement such as the characteristics of the road surface, width and breadth of lanes, appropriate traffic signs. Legally enforceable measures is a law enforced on individual to practice while driving. Car and road user needs to comply with traffic laws in order to be save. Such as speed limit point for each type of vehicle according to road conditions. Educational training measures. It is a social extension process where everyone in the society are given the proper Knowledge in prevention from accident. The knowledge giving process can be joined together with academic. Different traffic campaigns such as using media as a medium for educating or collaborating with people in the community. Training Measures can be arranged both in the education system and in non-formal education system. Formal educational system is a way of creating awareness of consciousness in them to enable them use the road safely. By inserting this moral into the educational system at all levels from Kindergarten to High School. Informal education system is a method of knowledge dissemination and the mental building of traffic safety by using media such as press conferences, advertising, posters, leaflets, articles, as well as training programs, campaign for Safe Driving and Accident Prevention, How to use the car properly etc.

From the above literature review, the researcher draw out the following conceptual framework 


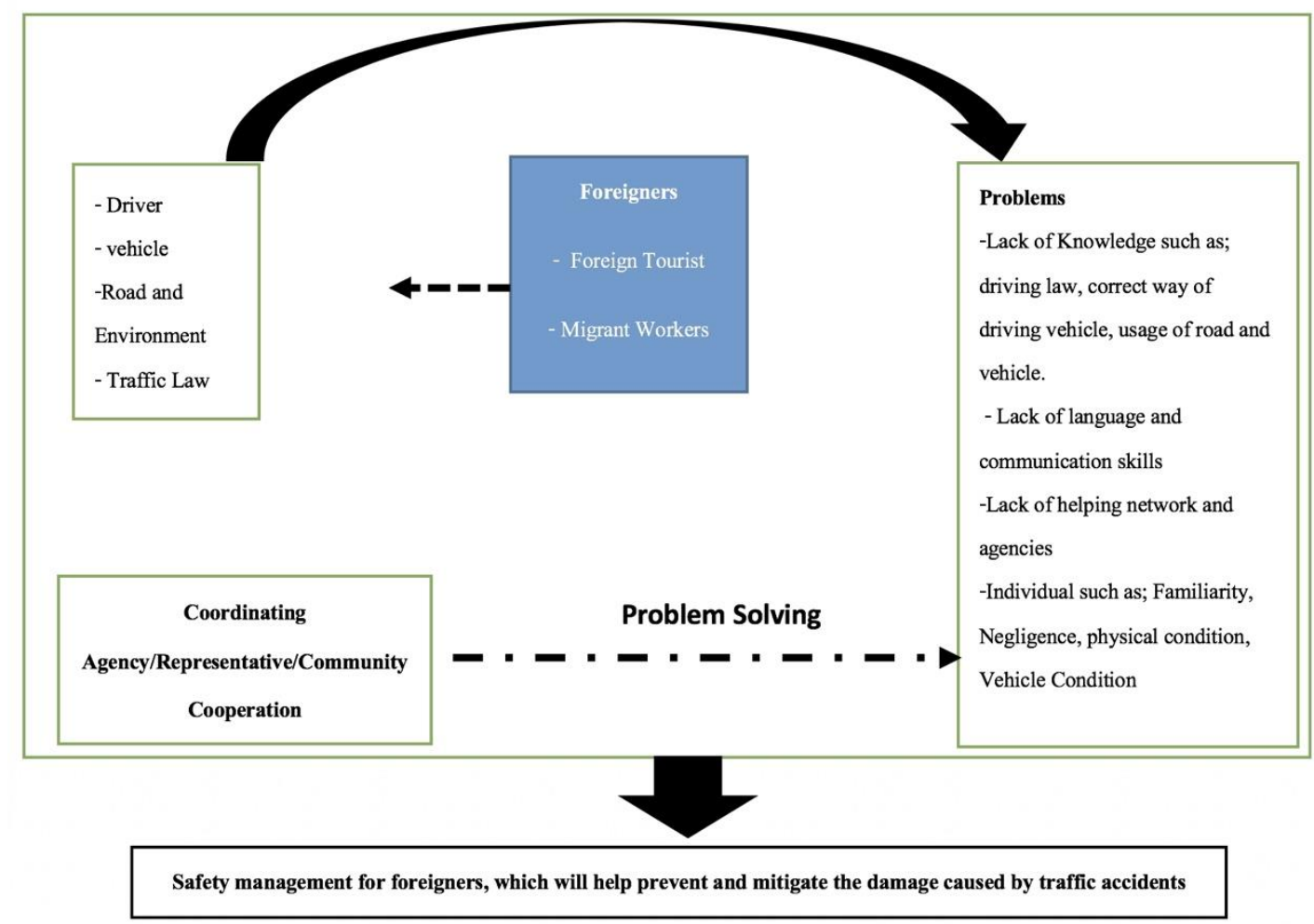

The conceptual framework shows problem analysis of traffic accident of foreigners according to the in dependent variables. However, solving these problems by relying on coordinating agencies/representative and co-operation from the community leads to effective and efficient safety management for foreigners, which will help prevent and mitigate the damage caused by traffic accidents.

Therefore, the researcher signify the importance of studying the details of road traffic accidents that involved foreigners. Looking into the causes the accidents, correct before and after traffic road accidents problems. However, the afore-mentioned information will be a guideline for safety management for foreigners, which will help prevent and mitigate the damage caused by traffic accidents.

\section{Methodology}

1. This study is research and development. Both qualitative and quantitative methods are been applied in this study

2. Qualitative research population incudes: ten people each from shops entrepreneurs, hotels, foreigners and people in Chiang Mai those involved with traffic safety management. Structured interviews was been used as a tool in gathering the required information. In order to study problem solution of traffic accidents before and after the traffic accident of foreigners and study the management approach to prevent and mitigate the damage caused by traffic accidents. In terms of population related to quantitative method, includes 236 traffic accident cases of foreigners between years 2014 - May 2017 in order to study the 
characteristics and cause of traffic accidents of foreigners. The tools used for quantitative method include recorded cases.

3. Data collection in this research work was been classified according to the research methodology by the researcher.

\section{Qualitative Method:}

Data collection by interview: The researcher used structured interview methods in collecting data from shops entrepreneurs, hotels, foreigners and people in Chiang Mai those involved with traffic safety management by specifically selecting them and the processed involved are as follows 1) The researcher introduce himself in order to starts data collection 2) Make the interviewee know the research purpose. 3) Proceeds on interview 4) gathered all interview questions and check for perfection, then proceeds to data analysis and it comprises of the following. 1) Check the completeness of the interview in order to possess all the specified information. 2) Separate interview responses in order to gather the frequency of safety management approaches for foreigners, prevent and mitigate the damage caused by traffic accidents.

\section{Quantitative Method:}

The collection of data were from the Chiang Mai police station records and Chiang Mai Tourist Police Station in Chiang Mai. Collection of data from recorded cases includes the following. 1) Identification letter from the university to the police station, which is the source of information was been given to the researcher to present to the station in order to requests for road accidents data during 2014 - 2017. 2) The researcher fill in required information in a case file. 3) Gathered all retrieved data and check for it's completeness and proceeds to analyzes. 4) The results were been analyzed and used to describe the characteristics and cause traffic accidents, explain the problem before and after the traffic accident of foreigners

4. Data analyzes in this research work was been classified according to the research methodology by the researcher.

Qualitative Data: The researcher uses content analysis method by collecting from the relevant research papers, interviews and they were been presented by descriptive analysis as follows:

Analysis of interview data: The researcher conducted analysis after gathering required information by choosing content analysis on the gathered data and it comprises of the following 1) Collect data obtained from the interview. The interview issues include approach to safety management for foreigners in order to prevent and mitigate the damage caused by traffic accidents. 2) Classify all data according to the study scope and 3) gather all retrieved information for guideline discussion on safety management for foreigners to prevent and mitigate the damage caused by traffic accidents. 


\section{Quantitative data has the following data analysis}

Information obtained from recorded cases are been analyzed in quantitative method, the researcher examined the data for correctness and accuracy of the case recorded. After that, the data were been saved into a data file in order to analyze and process them with computer application program.

5. Statistics used in data analysis were basic statistics and percentage

\section{Result and Conclusion}

Statistics of traffic accidents of foreigners during 2014 - May 2017 are from the Chiang Mai police station records and Chiang Mai Tourist Police Station in Chiang Mai. shows that there are 236 cases of traffic accidents and they are been explained explicitly below.

Traffic accidents, which involve foreigners during 2014 - 2017 shows that there were 169 cases accounted for $71.61 \%$. Accident victims that were male recorded 67 cases or $28.39 \%$. Accident victims that were female and has the highest rate of accident ranges between ages 25 40 years and it comprises of 140 cases or $59.32 \%$, followed by age 41 and above with 60 cases or $25.42 \%$, and below age 25 years with a total of 36 cases respectively at $15.26 \%$. Majority of the nationality that get involved in traffic accidents is Myanmar with 117 cases, $49.58 \%$ followed by Chinese 66 cases, 27.97\%, European victims 51 cases at 21.61\%, Laos and Cambodia 1 case at $0.42 \%$.

The characteristic and causes of traffic accidents showed that there were 236 driving behavior accounted for $100 \%$ were due to negligence, Road conditions showed that road condition were found to be in normal condition at 181 cases at $76.70 \%$. Safety warning 38 cases at $16.10 \%$ and there were 17 cases from the construction/damage roads, at 7.2\%. Apart from this, Most of the accidents occurs at U-turns with 106 cases at $44.91 \%$, followed by intersections with 46 cases at $19.50 \%$ and straight roads with 43 cases at $18.22 \%$ respectively. Redirecting roads with 41 cases at $17.37 \%$. In terms of time when the accident occurs, shows that most of the time was during the daytime ( $6.00-18.00 \mathrm{hrs}$.) with 134 cases at $56.78 \%$ and during the night time was between (18.01 - 5.59hrs) with 102 cases at $43.22 \%$. In terms of damaged caused showed that, most of the time it resulted to injuries/ loss of property with 193 cases at $81.78 \%$ and loss of lives occurs in 43 cases at $18.22 \%$. In terms of law pursuit shows that, 205 cases at $86.87 \%$ were been charged to court and 31 cases escaped after the accident at $13.13 \%$ and those that cannot be arrested during the accident 194 cases but were reported within 1-3 hours after the accident at $82.20 \%$ and 42 cases at $17.80 \%$ were reported within one hour after the accident.

In terms of problem of both before and after traffic accident of foreigners showed that, most of the people knows basic information that needs to be done such as contacting related agencies after an accident, but they still lack the understanding, lack effective coordination in order to get help. After a traffic accident with foreigners, related officers embark on investigating their legal entry into the kingdom, by checking their identification and passports. Prosecution process went according to road traffic laws. The accident were reported within 1-3 hours after it occurred, which 
however, shows there are delays in terms of notifications and prosecution process of occurred accident. However, this might leads to the distortion of such cases, resulting to injustice in prosecution process. The aforementioned delays were as a result that foreigners involved in the accident do not know how and what to do after such accidents. Because they only know basic information, but they lack practical understanding such as not knowing where to report the incident, not knowing what number to call after an accident in a particular area. Including language communication barrier of between officers and foreigners.

Guidelines for the prevention and mitigation of damage caused by traffic accidents of foreigners found that, apart from roads and environment, legal enforcement and the creation of community service. There should be continuous operation; especially Information, educate them on the uses of road and services, including the participation of related agencies or individuals in educating the people to know and understand the rules, laws and regulations. In collaboration with local organizations, citizens and local communities, such as: giving information on risk point, serves as a traffic volunteer, serves as a mediator between a local resident and officer.

Therefore, guidelines and approach to prevent and mitigate the damage caused by traffic accidents of foreigners from this research discovered that, corporation from both drivers, vehicles roads and environment including traffic laws should be enforced on drivers to strictly follow the law. The law should be updated to reflect current conditions and there should be serious punishment for violators. Including community participation in form of a center stage for coordination or representative in helping to create understanding in practice of foreigners. They should also help in communicating and giving knowledge to foreigners when an accident occurs, and they can be a representative in terms of negotiation, so that justice will be given to foreigners.

\section{Discussion}

According to the study, it shows that the characteristics and causes of traffic accidents that involved foreigners between 2014 - 2017 are mostly male aged between 25 - 40 years. They are Asian (Chinese) and Burmese migrant (Myanmar). This went in accordance with Chiang Mai Labor Office (2017) where they mentioned that Myanmar migrant workers has the highest number of population at 44,515 people. This was been gathered from first quarter survey of 2017. Majority of accidents occurred during the day between $6.00 \mathrm{am}$ and $6.00 \mathrm{pm}$. This was because tourists uses the said time in visiting natural tourist and attraction sites. However, this time was also the period when foreign migrant workers go to work. Moreover, the damaged and losses that occurred are in high rate. This went in accordance with statistics of casualties and traffic fatalities in Chiang Mai (January to October 2017), that shows that the rate of accident up to 33,547 accidents, in the aforementioned numbers 365 people died and 33,182 were injured. (Accident Information Center for Strengthening the Culture of Road Safety, 2017). Apart from that, it was also revealed that the main cause of accident are from personal driving habits filled with carelessness and inability of not knowing traffic rules and regulations. According to the Health Promotion Fund (2016) said, the severity of the accident is due to negligence and not wearing helmet and belts while driving of both drivers and passengers. This also went in accordance with World Health Organization (2016) 
which said, drivers need better understanding of how to use the road, road sign, use of vehicle and the usage of different lanes on the road.

In terms of problem of both before and after traffic accident of foreigners showed that, most of the foreigner knows basic information that needs to be done such as contacting related agencies after an accident, but they still lack the understanding. After a traffic accident with foreigners, related officers embark on investigating their legal entry into the kingdom, by checking their identification and passports. Prosecution process went according to road traffic laws. The accident were reported within 1-3 hours after it occurred, which however, shows there are delays in terms of notifications and prosecution process of occurred accident. However, this might leads to the distortion of such cases, resulting to injustice in prosecution process. The aforementioned delays were as a result that foreigners involved in the accident do not know where to report the accident, including communication barrier between officers and foreigners. Therefore, solving of problem both before and after accidents should start with basic training. Because, this serves as the starting point for knowledge, understanding and acceptance of other measures, such as the condition and use of vehicles, routes and different environments, how to drive, how to drive correctly according to traffic rules and driving etiquette.

Guidelines to prevent and mitigate the damage caused by traffic accidents of foreigners should corporates with both drivers, vehicles roads and environment. Traffic laws should be enforced on drivers to adhere strictly. The law should be updated to reflect current conditions and there should be serious punishment for violators. Including community participation in form of a center stage for coordination or representative in helping to create understanding in practice of foreigners. They should also help in communicating and giving knowledge to foreigners when an accident occurs, and they can be a representative in terms of negotiation, so that justice will be given to foreigners. The availability of center stage or representative to foreigners in terms of coordination when there is an accident will allow quick action to be in place so that the foreigners are been helped immediately.

\section{References}

Accident information center to strengthen road safety culture. (2017). Statistics of casualties and deaths chiang mai 2017. Retrieved from http://www.thairsc.com/p77/index.htm?provid=

Cabarkapa , M. and Avramovic , ZZ. (2017). Focus areas and measures to improve road traffic safety at the local level. Management, 7(2), 75-83 .

Chiang Mai Tourism and Sports Office. (2017). Tourism statistics. Retrieved from http://www.cmmots.com/news/view/1417592284.html

Chiang Mai Employment Agency. (2017). Number of Registered Burmese Migrant Workers for Year 2015. Chiang Mai: Chiang Mai Employment Office.

Chiang Mai Labor Office. (2017). The labour situation in Chiang Mai province. In Preparation for Asean Community. Retrieved from http://mol.go.th/sites/chiangmai.mol.go.th/files/sthaankaarnaerngngaancchanghwadechiiy ngaihm_1-60.pdf 
Department of Health and Promotion Foundation. (2016). Road Traffic Accident Report of Thailand 2012 - 2013. Bangkok: Office of Health Promotion Fund.

Interrisk Asia. (2017). Cause of accident. Retrieved from http://www.interriskthai.com/file_download/ 2016-06- 03 _ 16 _ 002 _Causes Factors_of_Road_Accident TH.pdf

Ministry of Tourism and Sports. (2013). Retrieved from http://www.mots.go.th/main.php?filename=index

Ministry of Tourism and Sports. (2017). Retrieved from http://www.mots.go.th/main.php?filename=index

Morley, A., Morris, A., Semaan, MA, Hancox , G. (2017). A guide for policy makers: on reducing road fatalities. Retrieved from https://www.pwc.com/m 1 / en / publications / road-safetypwc-guide-on-reducing-road-fatalities.pdf

National Statistical Office. (2017). Land traffic accident. Retrieved from http://service.nso.go.th/nso/web/statseries/statseries21.html

Office of Foreign Workers Administration Department of Employment. (2017). Facts that Needed to be Known for Myanmar, Laos and Cambodia Migrant Workers Working in Thailand. Bangkok: Department of Employment.

Office of Foreign Workers Administration. (2017). Working statistics of foreign workers. Retrieved from, https://www.doe.go.th/prd/alien/statistic/param/site/152/cat/82/sub/0/pull/module/view/li st-label

Thailand ranked second in world road accidents, millions are injured and six thousand disabled per year (2017). Retrieved from https://www.khaosod.co.th/monitor-news/news_267369

Thailand ' 2 nd on world road accident. (2016). Retrieved from http://www.thaihealth.or.th/Content/32580.

Tourism Authority of Thailand. (2017). Retrieved from http://thai.tourismthailand.org/

World Health Organization. (2016). World road safety situation report. Retrieved from http://www.who.int. 